\title{
ALTERAÇÕES HIDROELETROLÍTICAS E ACIDOBÁSICAS NO PÓS-OPERATÓRIO IMEDIATO DO TRANSPLANTE DE FÍGADO: ANÁLISE RETROSPECTIVA DE 20 ANOS EM CENTRO DE REFERÊNCIA NO NORDESTE DO BRASIL
}

\author{
Hydroelectrolytic and acid-basic alterations on immediate postoperative liver \\ transplantation: A 20-year retrospective analysis at a reference center in the \\ Northeastern Brazil
}

\author{
Olival Cirilo Lucena da Fonseca Neto ${ }^{1}$, Beatriz Costa Nava Martins ${ }^{2}$, Priscylla Jennie Monterio Rabêlo ${ }^{1}$, \\ Paulo Sérgio Vieira de Melo ${ }^{1}$, Gustavo Michel da Cunha Cruz ${ }^{1}$, Américo Gusmão Amorim , \\ Cláudio Moura Lacerda de Melo'
}

\begin{abstract}
RESUMO
Desde o desenvolvimento da técnica do transplante de fígado (TF) e da realização das primeiras cirurgias, os desafios em torno desse procedimento foram os mais variados. Distúrbios como acidose, hiponatremia, hipercalemia e hipocalcemia podem ser observados nos pacientes após TF. Apesar da recorrência, esses distúrbios são escassamente estudados. Objetivo: Analisar quais são as alterações hidroeletrolíticas e acidobásicas mais frequentes no pós-operatório imediato dos pacientes submetidos ao TF, sua prevalência e a relação dessas alterações com outras complicações frequentes nesses pacientes. Método: Este é um estudo retrospectivo transversal, que incluiu 599 pacientes que se submeteram ao transplante de fígado entre 1999 e 2021. A coleta dos dados foi realizada através da análise de prontuários da Unidade de Transplante de Fígado (UTF), do Hospital Universitário Oswaldo do Cruz em Recife (PE). Resultados: A hiponatremia esteve presente em 18\% da amostra, hipocalemia em $21 \%$, hipocalcemia em 77\%, 40\% apresentavam hiperfosfatemia e $69 \%$ possuíam hipomagnesemia. A hipoalbuminemia esteve presente em $98 \%$ dos pacientes e $69 \%$ possuíam hiperlactatemia. A acidose estava presente em mais de $55 \%$ da amostra. Conclusão: Os distúrbios hidroeletrolíticos e ácidobásicos são bastante prevalentes nos pacientes em pós-operatório imediato de transplante de fígado. Chamam atenção distúrbios do cálcio, magnésio, albumina, lactato, além da acidose.
\end{abstract}

Descritores: Desequilíbrio hidroeletrolítico; Desequilíbrio ácido base; Transplante de fígado.

Instituições:

1 Unidade de Transplante de Fígado, Hospital Oswaldo do Cruz, Recife, Pernambuco, Brasil

2 Faculdade de Ciências Médicas, Hospital Oswaldo do Cruz, Recife, Pernambuco, Brasil

https://doi.org/10.53855/bjt.v24i4.431

Correspondência:

Olival Cirilo Lucena da Fonseca Neto,

Email: olivalneto@globo.com

Recebido em: 19/08/2021

Aceito em: 21/09/2021

\section{INTRODUÇÃO}

Desde o desenvolvimento da técnica de transplante de fígado (TF) e da realização das primeiras cirurgias, os desafios em torno desse procedimento foram os mais variados. ${ }^{1}$ Tratamento de escolha para pacientes com doença hepática crônica em estado terminal, o TF, apresenta complicações frequentes como disfunção precoce do enxerto, trombose e alterações arteriais, complicações biliares e outras. No pós-operatório imediato do TF, porém, duas complicações chamam atenção pela instabilidade proporcionada ao paciente e pela fragilidade de desenvolvimento: os distúrbios hidroeletrolíticos e ácido-básicos. ${ }^{2}$ 
Alterações hidroeletrolíticas e acidobásicas no pós-operatório imediato do transplante de fígado: análise retrospectiva de 20 anos em centro de referência no Nordeste do Brasil

Devido ao ambiente de múltiplos fatores associados, o perfil fisiopatológico pré-operatório, administração de fluidos no perioperatório, reperfusão hepática e o estado pós-operatório, as mudanças nos eletrólitos e no padrão ácidobásico são bastante comuns. ${ }^{3}$ Distúrbios como acidose, hiponatremia, hipercalemia e hipocalcemia podem ser observados nos pacientes após TF. ${ }^{4}$ Apesar da recorrência, esses distúrbios são escassamente estudados.

Devido a isso, o objetivo do estudo foi analisar quais são as alterações hidroeletrolíticas e acidobásicas mais frequentes no pós-operatório imediato dos pacientes submetidos ao TF, a prevalência delas e a relação dessas alterações com outras complicações frequentes nesses pacientes.

\section{MÉTODOS}

Este é um estudo retrospectivo transversal, que incluiu 599 pacientes que se submeteram ao transplante de fígado, entre 1999 e 2021. A coleta dos dados foi realizada através de análise de prontuários na Unidade de Transplante de Fígado (UTF), localizada no Hospital Universitário Oswaldo do Cruz, em Recife (PE). Dentre os critérios de elegibilidade dos pacientes, foram incluídos: realização prévia de transplante hepático, idade mínima de 12 anos para enquadramento no escore MELD e apresentação em seu prontuário do documento com as informações da cirurgia, com preenchimento de dados e variáveis necessárias para análise, junto ao fluxograma dos exames. Pacientes foram excluídos das análises caso não tivessem em seus prontuários a maioria das variáveis do pós-operatório imediato disponíveis para análise.

O tamanho amostral foi determinado por conveniência, a partir da quantidade de prontuários disponíveis na UTF para análise.

Inicialmente, foi realizada uma análise descritiva numérica categórica e contínua dos dados para compor o perfil demográfico da população receptora dos transplantes, incluindo: sexo, idade, IMC, tipo sanguíneo, etiologia da doença hepática, comorbidades (HAS, DM, obesidade), uso de diuréticos, escore MELD e classificação quanto à urgência ou eletividade do transplante. Informações sobre a população dos doadores também foram coletadas quanto ao doador ser falecido ou vivo e sua classificação quanto a doador limítrofe para doação ou standard (padrão). O doador que não apresenta qualquer restrição na avaliação para doação apresenta característica padrão em conformidade para doação do órgão ao transplante.

Sequencialmente, foi realizada uma análise descritiva numérica categórica dos dados da população receptora dos transplantes, quanto às variáveis do pós-operatório imediato: $\mathrm{Na}$ (sódio), K (potássio), Ca (cálcio), P (fósforo), Mg (magnésio), dados de gasometria: $\mathrm{pH}$ sanguíneo, pCO2, pO2, HCO3 (bicarbonato), FiO2 (fração inspirada de oxigênio), SatO2 (saturação de oxigênio), BE (base excesso), além dos níveis de lactato, creatinina, ureia, albumina e amilase, e informação sobre o transplante ter cursado com síndrome de reperfusão ou não.

Os dados foram comparados com seus valores de referência previamente definidos. Baseando-se nessa análise, foi possível identificar as principais alterações eletrolíticas e acidobásicas nesses pacientes e suas frequências, assim como inferir algumas relações entre variáveis.

\section{Análise estatística}

As variáveis categóricas foram descritas por frequências absolutas e relativas. As variáveis contínuas foram descritas pela mediana e quartis, dada sua distribuição não normal. As prevalências das alterações laboratoriais, hidroeletrolíticas e acidobásicas foram calculadas para os dados completos e após imputação múltipla dos dados perdidos e apresentadas acompanhadas dos respectivos intervalos de confiança de $95 \%$. As variáveis gasométricas apresentavam alto percentual de perdas $(>30 \%)$, razão pela qual não foram imputadas. Para a análise estatística inferencial e comparações de grupos foram utilizados testes qui-quadrado ou exato de Fisher para variáveis categóricas e o teste de Mann-Whitney para variáveis contínuas, conforme aplicabilidade.

Todos os testes foram bicaudados, e valores finais de p menores que 0,05 foram considerados significativos. Todas as análises foram conduzidas em linguagem $R(R$ Foundation for Statistical Computing, Vienna, Austria).

\section{RESULTADOS}

A caracterização da amostra dos 599 pacientes com idade, sexo, tipo sanguíneo, índice de massa corporal (IMC), condições prévias e tipo de transplante está disposta na Tabela 1. Além disso, as medianas das variáveis analisadas no pós-operatório constam na Tabela 1, junto com as complicações e o tipo de doador do transplante.

A prevalência das alterações hidroeletrolíticas, acidobásicas e laboratoriais no pós-operatório imediato foram incluídas na Tabela 2 e Tabela 2.1. Percebeuse a prevalência de hiponatremia em $18 \%$ da amostra, hipocalemia em $21 \%$ e hipocalcemia em $77 \%$. Cerca de $69 \%$ dos pacientes apresentavam elevados níveis de lactato, e quase $98 \%$ deles possuíam hipoalbuminemia. A creatinina de $22 \%$ deles estava alterada. Quanto aos 
parâmetros acidobásicos, 55\% apresentaram acidose, possivelmente metabólica, visto que $70 \%$ da amostra possuía valores de bicarbonato abaixo da referência e $38 \% \mathrm{PCO}_{2}$ também diminuída.

Tabela 1: Caracterização da amostra total

\begin{tabular}{|c|c|}
\hline Variáveis & $\mathbf{N}=599$ \\
\hline Idade (anos) & $54.0(43.0-62.0)$ \\
\hline Sexo masculino & $402(67 \%)$ \\
\hline \multicolumn{2}{|l|}{ Tipo sanguíneo } \\
\hline A- & $16(2.7 \%)$ \\
\hline$A+$ & $206(34 \%)$ \\
\hline$A B-$ & $4(0.7 \%)$ \\
\hline $\mathrm{AB}+$ & $23(3.8 \%)$ \\
\hline B- & $6(1.0 \%)$ \\
\hline $\mathrm{B}+$ & $76(13 \%)$ \\
\hline O- & $26(4.3 \%)$ \\
\hline $\mathrm{O}+$ & $241(40 \%)$ \\
\hline IMC & $25.4(22.8-28.0)$ \\
\hline \multicolumn{2}{|l|}{ Condições prévias } \\
\hline Obesidade & $92(17 \%)$ \\
\hline Hipertensão arterial & 169 (29\%) \\
\hline Diabetes & $141(24 \%)$ \\
\hline MELD & $18.0(13.0-22.0)$ \\
\hline Uso de diuréticos & 266 (46\%) \\
\hline \multicolumn{2}{|l|}{ Tipo de transplante } \\
\hline Urgência & 11 (1.9\%) \\
\hline Eletivo & $582(98 \%)$ \\
\hline \multicolumn{2}{|c|}{$\begin{array}{l}\text { Exames laboratoriais no pós- } \\
\text { operatório imediato }\end{array}$} \\
\hline Sódio (mEq/L) & $139.0(136.0-142.0)$ \\
\hline Potássio (mEq/L) & $4.0(3.6-4.5)$ \\
\hline Cálcio (mg/dL) & $7.8(7.1-8.4)$ \\
\hline Fósforo (mg/dL) & $4.3(3.6-5.2)$ \\
\hline Magnésio (mg/dL) & $1.5(1.3-1.7)$ \\
\hline Ureia (mg/dL) & $31.0(24.0-43.0)$ \\
\hline
\end{tabular}

\begin{tabular}{ll}
\hline Variáveis & $\mathbf{N}=599$ \\
\hline Creatinina (mg/dL) & $0.8(0.6-1.1)$ \\
Albumina (g/dL) & $2.0(1.7-2.4)$ \\
Lactato (mmol/L) & $3.1(2.1-4.7)$ \\
Amilase (U/L) & $40.0(30.0-61.0)$ \\
Ph plasmático & $7.33(7.27-7.40)$ \\
PCO2(mmHg) & $37.0(32.5-42.0)$ \\
PO2(mmHg) & $143.0(96.0-190.5)$ \\
HCO3(mmol/L) & $20.2(17.2-23.8)$ \\
FiO2 & $0.4(0.2-0.5)$ \\
Base-excess & $-6.4(-9.8--3.3)$ \\
Sat O2(\%) & $99(96-100)$ \\
Complicações & $66(51 \%)$ \\
\hline Mravidade & $201(41 \%)$ \\
\hline
\end{tabular}

Dados apresentados como mediana (p25-p75) ou n (\%)

Algumas das variáveis foram associadas para análise de alterações conjuntas, como foi o caso dos valores de creatinina e sódio, na busca por analisar insuficiência renal, detalhados na Tabela 3. Inferiu-se que, dos 130 (22\%) pacientes com creatinina alterada, $26(21,7 \%)$ deles apresentavam, em associação, alteração de sódio e hiponatremia.

As variáveis acidobásicas: $\mathrm{pH}$, bicarbonato e $\mathrm{PCO} 2$ foram associadas com lactato na Tabela 4. Observamos que dos 92 (55\%) pacientes com acidose, 72 (87,5\%) deles apresentavam, conjuntamente hiperlactatemia. Além do lactato, o bicarbonato estava diminuído em 64 $(87,7 \%)$ dos pacientes com acidose. A PCO2 diminuída dos $64(38,3 \%)$ da amostra coincidiu também em 25 $(27,2 \%)$ com acidose. 
Alterações hidroeletrolíticas e acidobásicas no pós-operatório imediato do transplante de fígado: análise retrospectiva de 20 anos em centro de referência no Nordeste do Brasil

Tabela 2: Prevalência de alterações hidroeletrolíticas, acidobásicas e laboratoriais no pós-operatório imediato após imputação múltipla

\begin{tabular}{|c|c|c|c|}
\hline Variáveis (Valores de referência) & $\mathbf{N}$ & Prevalência & IC 95\% \\
\hline Sódio (135-145 mEq/L) & 599 & & \\
\hline$<135$ & & $107(18 \%)$ & $15 \%-21 \%$ \\
\hline$>145$ & & $49(8.2 \%)$ & $6.0 \%-10 \%$ \\
\hline Cálcio (8.5-10.5 mg/dL) & 599 & & \\
\hline$<8.5$ & & $460(77 \%)$ & $73 \%-80 \%$ \\
\hline$>10.5$ & & $8(1.3 \%)$ & $0.4 \%-2.3 \%$ \\
\hline Fósforo (2.5-4.5 mg/dL) & 599 & & \\
\hline$<2.5$ & & $31(5.2 \%)$ & $3.4 \%-6.9 \%$ \\
\hline$>4.5$ & & 242 (40\%) & $36 \%-44 \%$ \\
\hline Magnésio (1.7-2.2 mg/dL) & 599 & & \\
\hline$<1.7$ & & 415 (69\%) & $66 \%-73 \%$ \\
\hline$>2.2$ & & $25(4.2 \%)$ & $2.6 \%-5.8 \%$ \\
\hline Lactato $(0.5-2.2 \mathrm{mmol} / \mathrm{L})$ & 599 & & \\
\hline$\leq 2.2$ & & $187(31 \%)$ & $28 \%-35 \%$ \\
\hline$>2.2$ & & 412 (69\%) & $65 \%-72 \%$ \\
\hline Ureia (10-50 mg/dL) & 556 & & \\
\hline$\leq 50$ & & $469(84 \%)$ & $81 \%-87 \%$ \\
\hline$>50$ & & $87(16 \%)$ & $13 \%-19 \%$ \\
\hline Creatinina (mg/dL) & 579 & & \\
\hline Normal & & $449(78 \%)$ & $74 \%-81 \%$ \\
\hline Aumentada * & & $130(22 \%)$ & $19 \%-26 \%$ \\
\hline Albumina (3.5-4.8 g/dL) & 456 & & \\
\hline$<3.5$ & & 447 (98\%) & $97 \%-99 \%$ \\
\hline$\geq 3.5$ & & $9(2.0 \%)$ & $0.7 \%-3.3 \%$ \\
\hline Amilase (28-100 U/L) & 489 & & \\
\hline$\leq 100$ & & 444 (91\%) & $88 \%-93 \%$ \\
\hline$>100$ & & 45 (9.2\%) & $6.6 \%-12 \%$ \\
\hline
\end{tabular}

*Homens: 0,7-1,2 mg/Dl; Mulheres: 0,5-0,9 $\mathrm{mg} / \mathrm{dL}$ 
Olival Cirilo Lucena da Fonseca Neto, Beatriz Costa Nava Martins, Priscylla Jennie Monterio Rabêlo, Paulo Sérgio Vieira de Melo, Gustavo Michel da Cunha Cruz, Américo Gusmão Amorim, Cláudio Moura Lacerda de Melo

Tabela 2.1: Prevalência de alterações hidroeletrolíticas, acidobásicas e laboratoriais no pós-operatório imediato

\begin{tabular}{lccc}
\hline \multicolumn{1}{c}{ Variáveis (Valores de referência) } & $\mathbf{N}$ & Prevalência & IC 95\% \\
\hline $\begin{array}{l}\text { PH plasmático (7.35-7.45) } \\
<7.35\end{array}$ & 166 & $92(55 \%)$ & $48 \%-63 \%$ \\
$>7.45$ & 167 & $19(11 \%)$ & $6.6 \%-16 \%$ \\
PCO2 (35-45 mmHg) & & & $31 \%-46 \%$ \\
$<35$ & & $64(38 \%)$ & $7.1 \%-17 \%$ \\
$>45$ & 167 & $20(12 \%)$ & $8.5 \%-19 \%$ \\
PO2 (80-100 mmHg) & & $23(14 \%)$ & $66 \%-80 \%$ \\
$<80$ & & $122(73 \%)$ & \\
$>100$ & 121 & & $62 \%-78 \%$ \\
Bicarbonato (22-26 mmol/L) & & $85(70 \%)$ & - \\
$<22$ & & $0(0 \%)$ & $70 \%-83 \%$ \\
$>26$ & 160 & $122(76 \%)$ & $0.4 \%-5.8 \%$ \\
Base excess (-3 - 3 mmol/L) & & $5(3.1 \%)$ & \\
$<-3$ & & & \\
$>3$ & & & \\
\hline
\end{tabular}

Tabela 3: Associação entre alterações da creatinina e níveis de sódio plasmático

\begin{tabular}{lllll}
\hline & & \multicolumn{2}{c}{ Creatinina } & Valor- $\mathbf{p}^{1}$ \\
& & \multicolumn{1}{c}{ Normal (449) } & Alterada (130) & \\
\hline Sódio (mEq/L) & $139.0(136.0-142.0)$ & $139.0(136.0-142.0)$ & $139.0(135.0-141.0)$ & 0.14 \\
$<135$ & $102(18.2 \%)$ & $76(17.2 \%)$ & $26(21.7 \%)$ & 0.51 \\
$135-145$ & $414(73.8 \%)$ & $330(74.8 \%)$ & $84(70.0 \%)$ & \\
$>145$ & $45(8.0 \%)$ & $35(7.9 \%)$ & $10(8.3 \%)$ & \\
\hline
\end{tabular}

Dados apresentados como mediana (p25-p75) ou $n(\%)$.

IWilcoxon rank sum test; Pearson's Chi-squared test 
Alterações hidroeletroliticas e acidobásicas no pós-operatório imediato do transplante de fígado: análise retrospectiva de 20 anos em centro de referência no Nordeste do Brasil

Tabela 4: Associação entre alterações do pH sérico e níveis de lactato, bicarbonato e PCO2

\begin{tabular}{|c|c|c|c|c|c|}
\hline \multirow{2}{*}{ Variáveis } & \multirow{2}{*}{ Total } & \multicolumn{3}{|c|}{$\mathrm{pH}$} & \multirow{2}{*}{ Valor-p ${ }^{1}$} \\
\hline & & Acidose (92) & Normal (55) & Alcalose (20) & \\
\hline \multirow[t]{2}{*}{ Lactato (mmol/L) } & $3.6(2.4-5.2)$ & $4.1(2.8-6.1)$ & $3.3(2.2-5.0)$ & $2.5(2.2-4.4)$ & 0.037 \\
\hline & & & & & 0.18 \\
\hline$\leq 2.2$ & 31 (21.4\%) & $14(16.3 \%)$ & $13(29.5 \%)$ & 4 (26.7\%) & \\
\hline$>2.2$ & 114 (78.6\%) & 72 (83.7\%) & 31 (70.5\%) & $11(73.3 \%)$ & \\
\hline \multirow[t]{2}{*}{$\begin{array}{l}\text { Bicarbonato } \\
\text { (mmol/L) }\end{array}$} & $20.2(17.1$ - 23.9) & $18.0(15.2-20.3)$ & $23.0(20.2-24.8)$ & $26.0(24.0-28.2)$ & $<0.001$ \\
\hline & & & & & $<0.001$ \\
\hline$<22$ & $82(62.6 \%)$ & 64 (87.7\%) & 16 (38.1\%) & 2 (12.5\%) & \\
\hline $22-26$ & $36(27.5 \%)$ & 8 (11.0\%) & $21(50.0 \%)$ & 7 (43.8\%) & \\
\hline$>26$ & 13 (9.9\%) & $1(1.4 \%)$ & 5 (11.9\%) & $7(43.8 \%)$ & \\
\hline \multirow[t]{2}{*}{$\mathrm{PCO}_{2}(\mathrm{mmHg})$} & $37.0(32.5-42.0)$ & $39.0(34.2-44.0)$ & $35.0(29.5-40.0)$ & $34.0(27.0-38.2)$ & $<0.001$ \\
\hline & & & & & $<0.001$ \\
\hline$<35$ & $64(38.3 \%)$ & $25(27.2 \%)$ & 27 (49.1\%) & $12(60.0 \%)$ & \\
\hline $35-45$ & $83(49.7 \%)$ & 49 (53.3\%) & 27 (49.1\%) & 7 (35.0\%) & \\
\hline$>45$ & 20 (12.0\%) & 18 (19.6\%) & $1(1.8 \%)$ & $1(5.0 \%)$ & \\
\hline
\end{tabular}

Dados apresentados como mediana (p25-p75) ou n (\%).

${ }^{1}$ Kruskal-Wallis rank sum test; Fisher's exact test

\section{DISCUSSÃO}

A diminuição dos níveis de sódio, hiponatremia, em pacientes elegíveis ao TF no pré e pós-operatório da cirurgia foi associada a consequências como alterações neurológicas e renais e aumento de mortalidade. ${ }^{5} \mathrm{Em}$ nosso estudo, entre as alterações nos níveis de sódio no pós-operatório imediato do TF, como mostra a Tabela 2, foi predominante a hiponatremia, presente em 107 (18\%) pacientes.

Possivelmente, o desenvolvimento da hiponatremia tem relação com a infusão de substâncias no peri-operatório, como cristaloides e plasma, o que rapidamente pode alterar os valores desse eletrólito. É válido lembrar que apesar disso, a hiponatremia possui etiologia baseada em fatores múltiplos. ${ }^{6}$ Em contrapartida, a presença de hipernatremia foi restrita a $8,2 \%$ da amostra.
Como discutido anteriormente, uma das possíveis implicações da hiponatremia são distúrbios renais; por isso, na tabela 3 foi associada a relação entre os valores de sódio e creatinina. A creatinina encontrou-se alterada em quase $22 \%$ da amostra, e sabe-se que dos $102(18,2 \%)$ pacientes da amostra com hiponatremia, $26(25,4 \%)$ deles apresentavam também alteração de creatinina. Sabe-se que um dos parâmetros do escore MELD (Model for End-stage Liver Disease) são os níveis de creatinina, sendo esse escore um fator prognóstico de desenvolvimento de insuficiência renal aguda (IRA). ${ }^{7}$ Quanto à IRA, há estimativa de que ao menos metade dos pacientes após o TF desenvolvem IRA. Os parâmetros de creatinina e hiponatremia são sinais de alerta para possível desenvolvimento de insuficiência renal nesses pacientes após TF. ${ }^{8}$ Uma alteração que também pode sugerir insuficiência renal é a uremia, que está presente em 85 (16\%) pacientes da amostra. 
Olival Cirilo Lucena da Fonseca Neto, Beatriz Costa Nava Martins, Priscylla Jennie Monterio Rabêlo, Paulo Sérgio Vieira de Melo, Gustavo Michel da Cunha Cruz, Américo Gusmão Amorim, Cláudio Moura Lacerda de Melo

Os distúrbios acidobásicos são também muito comuns tanto em pacientes em pré-operatório quanto no pósoperatório imediato. Segundo Boniatti (2013), em estudo feito com 52 pacientes admitidos em UTI após TF, 37 (71\%) deles apresentaram acidose metabólica no pós-operatório imediato. ${ }^{3}$ Dando seguimento a essa linha, em nossa amostra, 92 (55\%) pacientes apresentaram acidose no pós-operatório imediato, com $64(70 \%)$ apresentando valores de bicarbonato abaixo do limite inferior, sugerindo uma prevalência de acidose metabólica. A PCO2 apresentou-se acima do limite superior da referência em $20 \%$ dos pacientes que já apresentavam acidose (indicando uma tentativa de compensação dessa acidose) e $50 \%$ dos pacientes com acidose apresentavam valores de PCO2 normais. No estudo de Story (2008), há uma sugestão de que a acidose seja causada por elevação dos níveis de cloro e de ânions não mensurados no plasma. Concomitante com a hipercloremia, a hiponatremia auxiliaria no desenvolvimento desse distúrbio acidobásico. ${ }^{9}$ Existem hipóteses de que possíveis causas para a acidose metabólica com hipercloremia e hiponatremia sejam a administração de soluções com cloro durante a cirurgia e também o estado pré-cirúrgico do paciente. ${ }^{3}$

O lactato é um eletrólito importante, que pode alertar a partir da sua elevação, várias alterações metabólicas, tais como um estado de hipoperfusão, de hipóxia e de acidose. Em pacientes críticos, é um sinal de pior prognóstico. $^{10}$ Quando se trata do pós-operatório imediato do TF, o lactato pode indicar possível disfunção do enxerto hepático, além de ser um indicador de mortalidade desses pacientes. ${ }^{11}$ Em nossa amostra, cerca de 412 (70\%) dos pacientes apresentavam valores de lactato acima da referência. Dos pacientes que apresentavam acidose, $78 \%$ deles apresentavam concomitantemente hiperlactatemia, confirmando a relação entre elas. Em outro estudo que quantificava os valores pós-operatórios de lactato no TF, cerca de $68.75 \%$ da amostra apresentavam valores de lactato acima da referência proposta, coincidindo com os valores aqui encontrados. ${ }^{11}$

A alteração de albumina em nossa amostra foi a mais consistente: 447 (98\%) pacientes da amostra apresentaram hipoalbuminemia no pós-operatório imediato do TF. A albumina é uma proteína de extrema importância e a hipoalbuminemia já é um distúrbio clássico em pacientes graves. Desde a elegibilidade do paciente ao TF, valores diminuídos de albumina causam aumento do risco de mortalidade dos pacientes ainda na espera pelo transplante..$^{12}$ Alguns estudos já mostraram também as consequências da queda nos níveis dessa proteína após o TF, e uma delas foi a menor capacidade sistêmica de suporte ao transplante, além da interferência em possíveis complicações em UTI desses pacientes, que costumam ser bem maiores..$^{13,14}$ Em contrapartida, essa proteína tem efeito oposto ao da acidose nesses pacientes, atenuando esse distúrbio com seu padrão alcalinizante. ${ }^{9}$

Diferentemente do que a maioria dos estudos relata, $123(21 \%)$ pacientes da amostra apresentaram hipocalemia, contra $26(4,3 \%)$ que apresentaram hipercalemia. Os riscos da diminuição dos níveis de potássio nos pacientes após TF são, principalmente, os vômitos e a diarreia. Uma hipótese para apresentação de hipocalemia nesses pacientes pode ter acontecido em contrapartida a uma tentativa de neutralização dos níveis mais elevados de potássio, distúrbio mais comum pelo uso de soluções atenuantes, como as de glicose, bicarbonato ou cálcio. ${ }^{15}$

A hipocalcemia foi um distúrbio bastante presente em nossa amostra; 460 (77\%) pacientes apresentavam essa alteração. Esse distúrbio é frequente especialmente nos primeiros dias de pós-operatório, além do imediato. Possíveis hipóteses para essa alteração são o estado de resposta ao trauma vivenciado após o TF e mudanças renais em relação a excreção de eletrólitos. Além disso, o aumento de consumo e perda desse metabólito pode impactar em sua concentração. É importante atentar para possíveis complicações relacionadas à deficiência desse eletrólito, como alterações neuromusculares, cardiovasculares e outras. ${ }^{16}$

Outro distúrbio que apresentou elevada prevalência foi a hipomagnesemia, em cerca de 415 (70\%) pacientes da amostra. Esse eletrólito apresenta padrão de maior queda no pós-operatório imediato, com posterior melhora de parâmetros. Alguns estudos afirmam que mesmo após a administração desse eletrólito, seus níveis apresentam tendência de permanecer em queda. Seus valores parecem apresentar diminuição ainda durante o peri-operatório e a maior hipótese de sua origem é o estado pós-cirúrgico.

A presença de hiperfosfatemia em 242 (40\%) pacientes da nossa amostra chamou atenção, visto que a maior parte dos estudos sobre esse eletrólito em pós-operatório de TF mostram predominância de hipofosfatemia. A primeira hipótese para explicar esse aumento do fósforo seria uma alteração renal com queda na excreção, porém a hipótese mais forte é o menor consumo inicial desse eletrólito pelo corpo. É fundamental atentar para esse distúrbio eletrolítico, pois ele já foi associado à disfunção precoce do 
Alterações hidroeletroliticas e acidobásicas no pós-operatório imediato do transplante de fígado: análise retrospectiva de 20 anos em centro de referência no Nordeste do Brasil

enxerto, visto que esses pacientes apresentavam hiperfosfatemia no pós-operatório imediato. ${ }^{17}$ Além disso, a hiperfosfatemia no pós-operatório imediato também foi associada à maior chance de desenvolver injúria renal aguda. ${ }^{18}$

Em uma análise clínica, as alterações da enzima amilase não se mostraram tão preponderantes, com mais de $90 \%$ da amostra apresentando níveis normais dessa enzima.

\section{CONCLUSÃO}

Percebe-se assim, que os distúrbios hidroeletrolíticos e ácido-básicos são bastante prevalentes nos pacientes em pós-operatório imediato de transplante de fígado. Predominam a hiponatremia, hipocalcemia, hipomagnesemia e hiperfosfatemia. A hipoalbuminemia como a alteração mais consistente desses pacientes junto com hiperlactatemia. O distúrbio ácido-básico dominante foi a acidose.

\section{ABSTRACT}

Since the development of the liver transplantation (LT) techniques and the first surgeries, challenges around this procedure emerged. Disorders such as hyponatremia, hyperkalemia and hypocalcemia may be observed in patients after LT. Despite the frequency, these disturbs are poorly studied. Purposes: To analyze which hydroelectrolytic disorders and acid-base are most frequent in the immediate postoperative of patients submitted to LT, their prevalence and relation of these alterations with other frequent complications in those patients. Methods: This is a transversal retrospective study that included 599 patients who underwent LT between 1999 and 2021. Data collection was performed by analyzing medical records in the Unidade de Transplante de Fígado (UTF), located at Universitary Hospital Oswaldo do Cruz in Recife (PE). Results: Hyponatremia was present in $18 \%$ of the sample, hypokalemia in $21 \%$, hypocalcemia in $77 \%, 40 \%$ presented hyperphosphatemia, and $69 \%$ had hypomagnesemia. Hypoalbuminemia was present in $98 \%$ of patients, and $69 \%$ had hyperlactatemia. Acidosis was present in above $55 \%$ of the sample. Conclusion: Hydroelectrolytic and acid-base disorders are quite prevalent in patients on the immediate postoperative of LT. Calcium, magnesium, albumin, lactate, and acidosis disorders are important and draw attention.

Keywords: Water Electrolyte Imbalance; Acid base Imbalance; Liver transplantation.

\section{Agradecimentos}

Agradecemos imensamente à Unidade de Transplante de Fígado e toda a sua equipe pela oportunidade e disponibilidade em permitir a realização deste estudo.

Agradecemos a todos que ajudaram e possibilitaram a concretização deste trabalho. 


\section{REFERÊNCIAS}

1. Meirelles Júnior RF erreir., Salvalaggio $P$, Rezende $M B$ run. de, Evangelista AS, Guardia BD ell., Matielo CE duard. L, et al. Liver transplantation: history, outcomes and perspectives. Einstein (Sao Paulo). 2015;13(1):149-52.

2. Amaral B, Vicente M, Pereira CSM, Araújo T, Ribeiro A, Pereira R, et al. Approach to the liver transplant early postoperative period: An institutional standpoint. Rev Bras Ter Intensiva. 2019;31(4):561-70.

3. Boniatti MM, Filho EMR, Cardoso PRC, Vieira SRR. Physicochemical evaluation of acid-base disorders after liver transplantation and the contribution from administered fluids. Transplant Proc [Internet]. 2013;45(6):22837. Available from: http://dx.doi.org/10.1016/j. transproceed.2013.03.044

4. Parekh KN, Crowley JC, Liu LL. Anesthesia and Perioperative Care for Organ Transplantation. Anesth Perioper Care Organ Transplant. 2017;

5. Karapanagiotou A, Kydona C, Papadopoulos S, Dimitriadis C, Giasnetsova T, Rempelakos G, et al. The effect of hyponatremia on the outcome of patients after orthotopic liver transplantation. Transplant Proc [Internet]. 2012;44(9):2724-6. Available from: http://dx.doi. org/10.1016/j.transproceed.2012.09.095

6. Faenza S, Bernardi E, Cuppini F, Gatta A, Lauro A, Mancini $E$, et al. Intensive care complications in liver and multivisceral transplantation. Transplant Proc. 2005;37(6):2618-21.

7. Feldkamp T, Bienholz A, Paul A, Saner FH. Renal damage after liver transplantation. Biosci Rep. 2020;40(1):1-11.

8. Durand F, Francoz C, Asrani SK, Khemichian S, Pham TA, Sung RS, et al. Acute Kidney Injury after Liver Transplantation. Transplantation. 2018;102(10):1636-49.

9. Story DA, Vaja R, Poustie SJ, McNicol L. Fencl-Stewart analysis of acid-base changes immediately after liver transplantation. Crit Care Resusc. 2008;10(1):19-23.

10. Basile-Filho A, Nicolini EA, Auxiliadora-Martins M, Silva $\mathrm{Jr} \mathrm{O}$ de $\mathrm{C}$ e. The use of perioperative serial blood lactate levels, the APACHE II and the postoperative MELD as predictors of early mortality after liver transplantation. Acta Cir Bras. 2011;26(6):535-40.
11. Jipa LN, Tomescu D, Droc G. The interrelation between arterial lactate levels and postoperative outcome following liver transplantation. Rom $\mathrm{J}$ Anaesth intensive care. 2014;21(2):106-12.

12. Ahn J, Sundaram V, Ayoub WS, Frenette C, Wong RJ. Hypoalbuminemia is associated with significantly higher liver transplant waitlist mortality and lower probability of receiving liver transplant. J Clin Gastroenterol. 2018;52(10):913-7.

13. Cohen J, Shapiro M, Grozovski E, Mor E, Shaharabani E, Shapira Z, et al. Should hypoalbuminemia after liver transplantation be corrected? Transplant Proc. 2001;33(6):2916-7.

14. Hiroi K, Matsusaki T, Kaku R, Umeda Y, Yagi T, Morimatsu H. Postoperative Course of Serum Albumin Levels and Organ Dysfunction After Liver Transplantation. Transplant Proc [Internet]. 2019;51(8):2750-4. Available from: https://doi. org/10.1016/j.transproceed.2019.01.199

15. Burlage LC, Hessels L, van Rijn R, Matton APM, Fujiyoshi M, van den Berg AP, et al. Opposite acute potassium and sodium shifts during transplantation of hypothermic machine perfused donor livers. Am J Transplant. 2019;19(4):1061-71.

16. Mahmoud AAA, El-Shaarawy AM, Mansour MA, Abdelhaq MM, Maher MA, Kamal AM. Perioperative calcium, magnesium, and phosphorus levels in live donors for liver transplant. Exp Clin Transplant. 2015;13(6):550-5.

17. Hong SH, Kwak JA, Jeon JY, Park CS. Prediction of early allograft dysfunction using serum phosphorus level in living donor liver transplantation. Transpl Int. 2013;26(4):402-10.

18. Hong SH, Park CO, Park CS. Prediction of newly developed acute renal failure using serum phosphorus concentrations after living-donor liver transplantation $\mathrm{J}$ Int Med Res. 2012;40(6):2199-212. 